\title{
Telescope Pupil Tracking using a Pyramid WFS
}

Jean-Pierre Véran, Glen Herriot

\author{
National Research Council Canada, 5071 W. Saanich Rd., Victoria, BC Canada V9E 2E7
}

\begin{abstract}
During an AO observation the Lyot stop of an infrared instrument fed by the AO system must remain aligned with the image of the telescope pupil. Uncertainties and drifts of the pupil image position must be accommodated by under-sizing the Lyot stop, which, especially for AO corrected observations, reduces very significantly the science productivity of the instrument. In the case of IRIS, the first client instrument of NFIRAOS on the TMT, there is a requirement to limit the under-sizing of the IRIS Lyot stop to $1 \%$ of the pupil diameter, which therefore means to tightly stabilize the image of the telescope pupil. In this paper, we show how this can be accomplished by finely aligning the IRIS Lyot stop to NFIRAOS during calibration (sighting actuators pokes on the NFIRAOS high-altitude DM with the IRIS pupil viewing camera), and, during observation, by processing the images obtained by the NFIRAOS Truth WFS to detect drifts and feeding this drift information to the telescope control system so it can repoint the beam fed to NFIRAOS. We show that this measurement of the pupil position with the Truth WFS can be accomplished in nominal observing conditions with an RMS error of less than $0.02 \%$ of the pupil diameter in bright time, and less than $0.03 \%$ of the pupil diameter in dark time. We include in this evaluation noise sources on the Truth WFS, as well as uncertainties on the reflectivity of the primary mirror segments due to their recoating schedule. This work directly feeds into the work presented in reference [1], which describes the full tolerance stack-up required to reach the $1 \%$ under-sizing of the Lyot stop.
\end{abstract}

Keywords: Adaptive optics, pupil centering, infrared astronomy

\section{INTRODUCTION}

NFIRAOS is the first light AO system being designed for the Thirty Meter Telescope (TMT). NFIRAOS can feed AO corrected near-infrared light to up to three different client instruments, including IRIS (InfraRed Imager and Spectrograph). Like all infrared astronomical instruments, IRIS has a cold Lyot stop on which the telescope pupil must be reimaged. The Lyot stop prevents non-stellar light from entering the instrument and creating an undesirable high background. The Lyot stop replicates the "features" of the telescope pupil, such as, in the case of TMT, the ragged edges of the primary mirror, as well as the secondary mirror support structures (spiders). Accurately centering the image of the telescope pupil onto the Lyot stop is absolutely critical, since centering errors will result in an unacceptable increase of background light. Many effects conspire to create centering errors, including calibration errors and opto-mechanical drifts within the instrument and within the telescope. In order to accommodate these residual centering errors, the Lyot mask is undersized, that is, it blocks more area than necessary, so that only stellar light will go through even in presence of pupil position errors. Under-sizing the mask however has the effect of reducing the effective aperture of the telescope. In addition, when the image is corrected by an $\mathrm{AO}$ system, reducing the effective aperture has also the effect of creating a broader point spread function (PSF), which further reduces the sensitivity of the telescope. Recent work has shown that for NFIRAOS and IRIS, under-sizing the Lyot mask by $1 \%$ of the pupil diameter increases the observing time required to achieve a given point source sensitivity by $30 \%$ ! [1]

Alignment of the IRIS Lyot stop with the TMT pupil is facilitated by a pupil-viewing camera (PVC). This camera however can only be deployed during calibration, and must be retracted during science observation. Therefore residual pupil drifts occurring after the PVC is retracted must be quantified, and the Lyot mask must be undersized accordingly. For IRIS, the requirement is that the Lyot stop should only be undersized by $1 \%$ of the pupil diameter. This is only possible with a tight alignment between NFIRAOS and IRIS, and a tight control of pupil position drifts between TMT and NFIRAOS.

A simplified sketch of the TMT-NFIRAOS-IRIS optical path is given in Figure 1: the telescope pupil is reimaged on the ground-conjugated deformable mirror (DM0) and then the infrared light is further relayed to the IRIS Lyot mask. A beamsplitter is used to send the visible light to a wavefront sensor (WFS). In the case of NFIRAOS, the WFS path as several WFSs, including six laser guide star (LGS) WFSs and one natural guide star (WFS), also called truth WFS 
(TWFS). The NFIRAOS TWFS is a pyramid WFS (PWFS), however for the purpose of this article, a Shack-Hartmann WFS (SHWFS) would work the same way.

Maintaining a tight alignment between the telescope pupil and the Lyot stop is decomposed into two main tasks: an offsky calibration task, using calibration sources within NFIRAOS, which aligns both the Lyot stop and the NGS WFS with DM0 (section 2); and, while observing, a feedback loop with the telescope control system that maintains a stable pupil image on the NGS WFS, and therefore onto the IRIS Lyot stop (section 3).

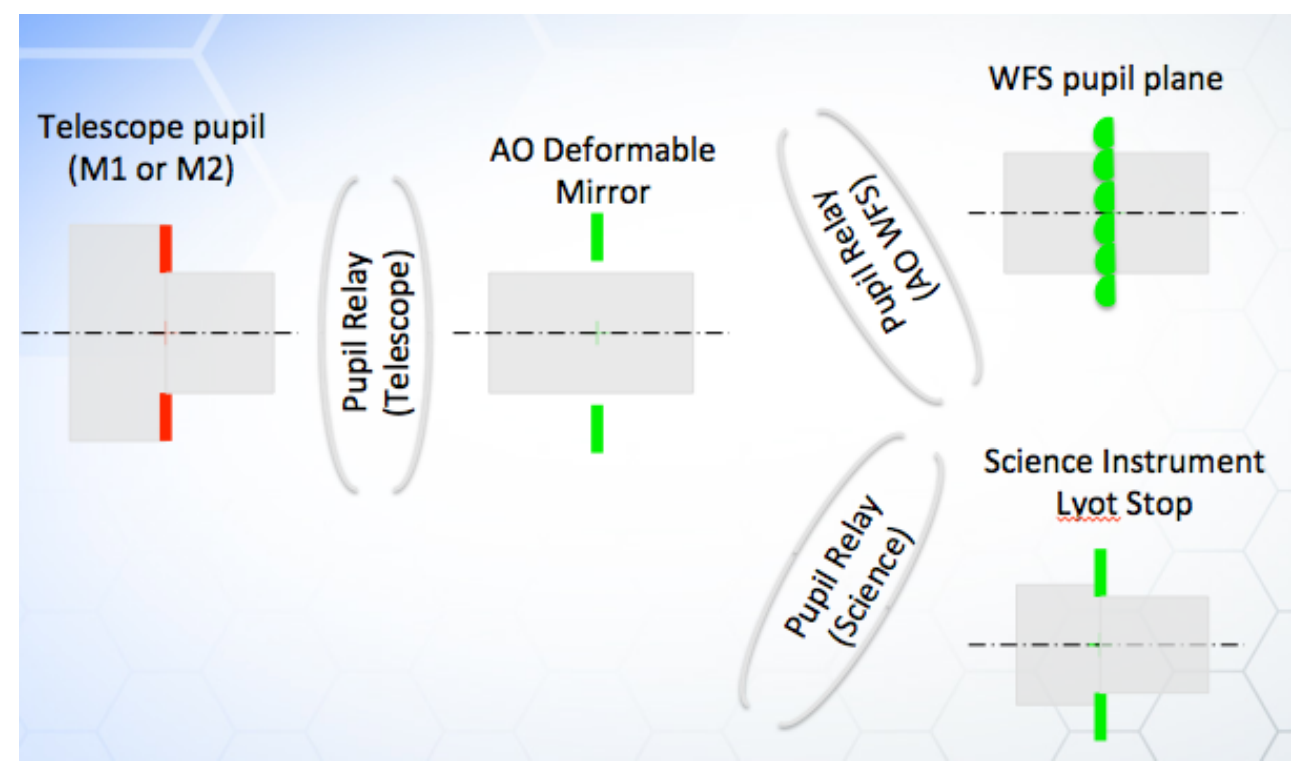

Figure 1: Generic sketch of the optical paths of a telescope-AO System-IR instrument such as TMT-NFIRAOS-IRIS

\section{ALIGNMENT DURING CALIBRATION}

\subsection{DM0 to NGS WFS alignment}

Aligning DM0 to the NGS WFS is a fairly well known procedure. It consists in illuminating DM0 with an artificial source, poking DM0 actuators and reading WFS measurements to find out the registration between DM0 and the WFS. Poked actuators produce a clear signature in the WFS signal that permits to locate them accurately. Then, using motorized optics, the pupil image is steered on the WFS until the registration is as desired. In NFIRAOS, the pupil can be steered on the WFS using a combination of stages carrying the WFS bench and a mirror close to the focal plane, able to tip, tilt and piston the beam. These mechanisms are specified in terms of accuracy and repeatability in such a way that the alignment can be ensured for all targets within the field of regard of the NGS WFS with a very high level of accuracy.

\subsection{DM0 to Lyot stop alignment}

Poking actuators on DM0 does not produce any signal on the IRIS PVC. However, poking actuators on DM11, the high altitude DM in NFIRAOS conjugated to $11.8 \mathrm{~km}$ above the telescope, does. This is because actuator pokes produce wavefront fluctuations at $11.8 \mathrm{~km}$, which, by Fresnel propagation, will create amplitude fluctuations in the pupil plane (at $0 \mathrm{~km})$. The effect, also known as Talbot effect, is maximized when using a waffle poke pattern. As shown in Figure 2, a modest $+/-0.8$ um poke pattern on DM11 results in a very clear intensity modulation on the PVC. By analyzing the recorded modulation pattern, the registration between DM11 and the PVC (and thus the Lyot stop) can be evaluated with a very high accuracy. The registration between DM11 and DM0 can be easily and accurately assessed using pokes on both DMs and measurements with the NGS WFS. Therefore this approach allows for a very accurate registration between DM0 and the IRIS Lyot stop. 

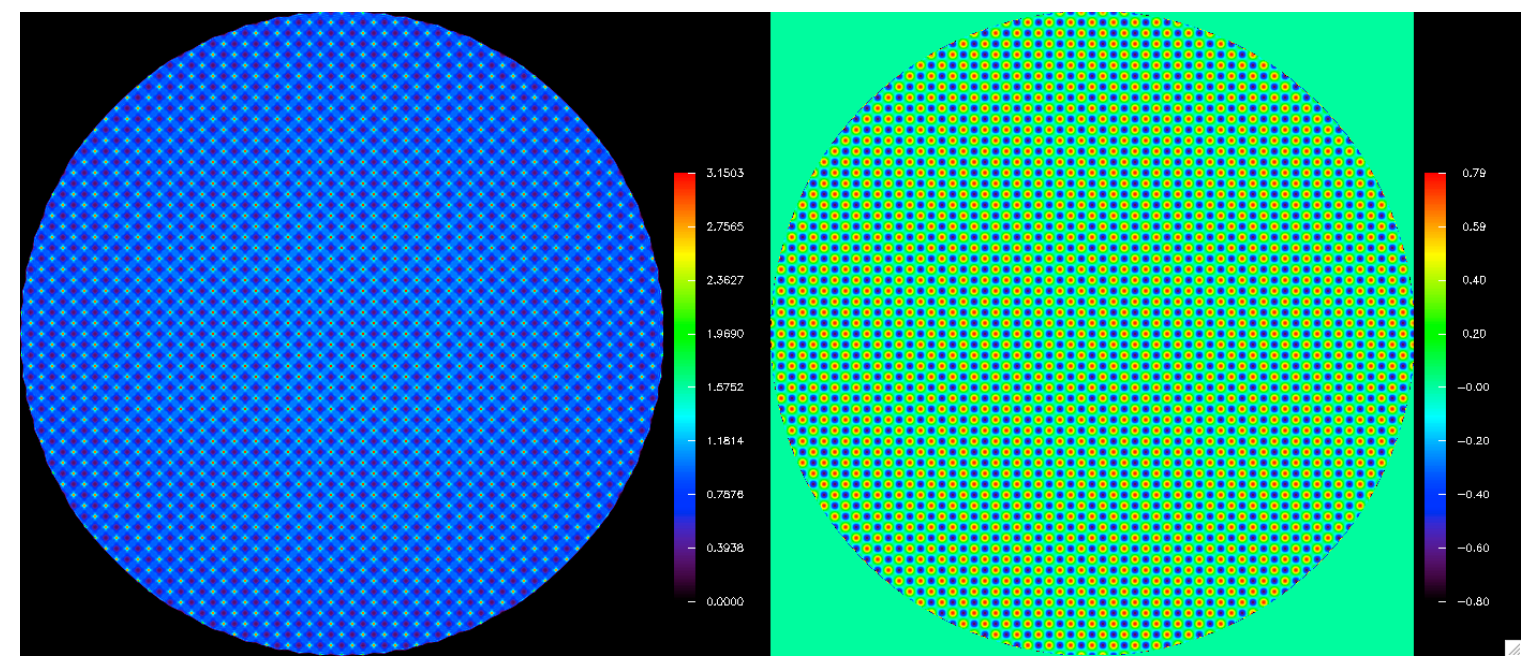

Figure 2: +/-0.8um waffle pattern on DM11 seen at the PVC. Left: intensity, which is recorded by the PVC. Right: wavefront error (in um)

\section{ON-SKY PUPIL STABILZATION ON LYOT STOP}

\subsection{General principle}

On-sky, the PVC can be used to check that the telescope pupil is properly imaged onto the Lyot stop. However, during a science observation, the PVC must be retracted. If the beam fed into NFIRAOS by the telescope drifts (for example the pointing of M3, which direct the beam to the Nasmith platform), then the telescope pupil will become misaligned on the Lyot stop (Figure 3). However, the same pupil drift will be seen on the Truth WFS. This drift can be detected from the Truth WFS measurements and fed back to the telescope control system, which can repoint the beam and cancel the drift.

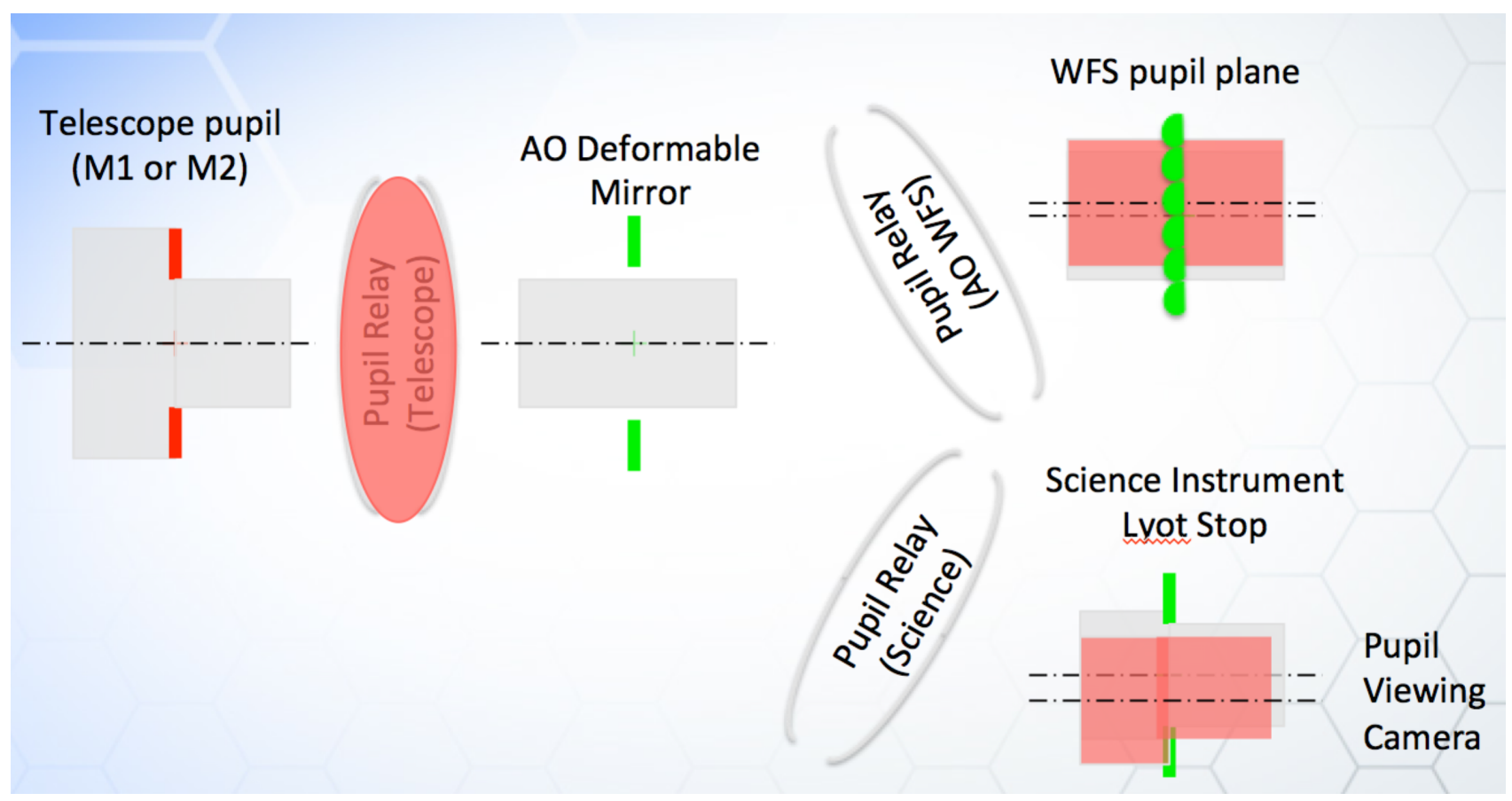

Figure 3: Effect of a telescope drift onto the Lyot stop and NGS WFS 


\subsection{TMT pupils}

Various TMT pupil models are shown at the top of Figure 4. The annular pupil on the left hand-side is used as reference. The two other images show a high fidelity representation of the TMT pupil, including segment gaps, ragged edges and M2 spiders, at two different position angles.
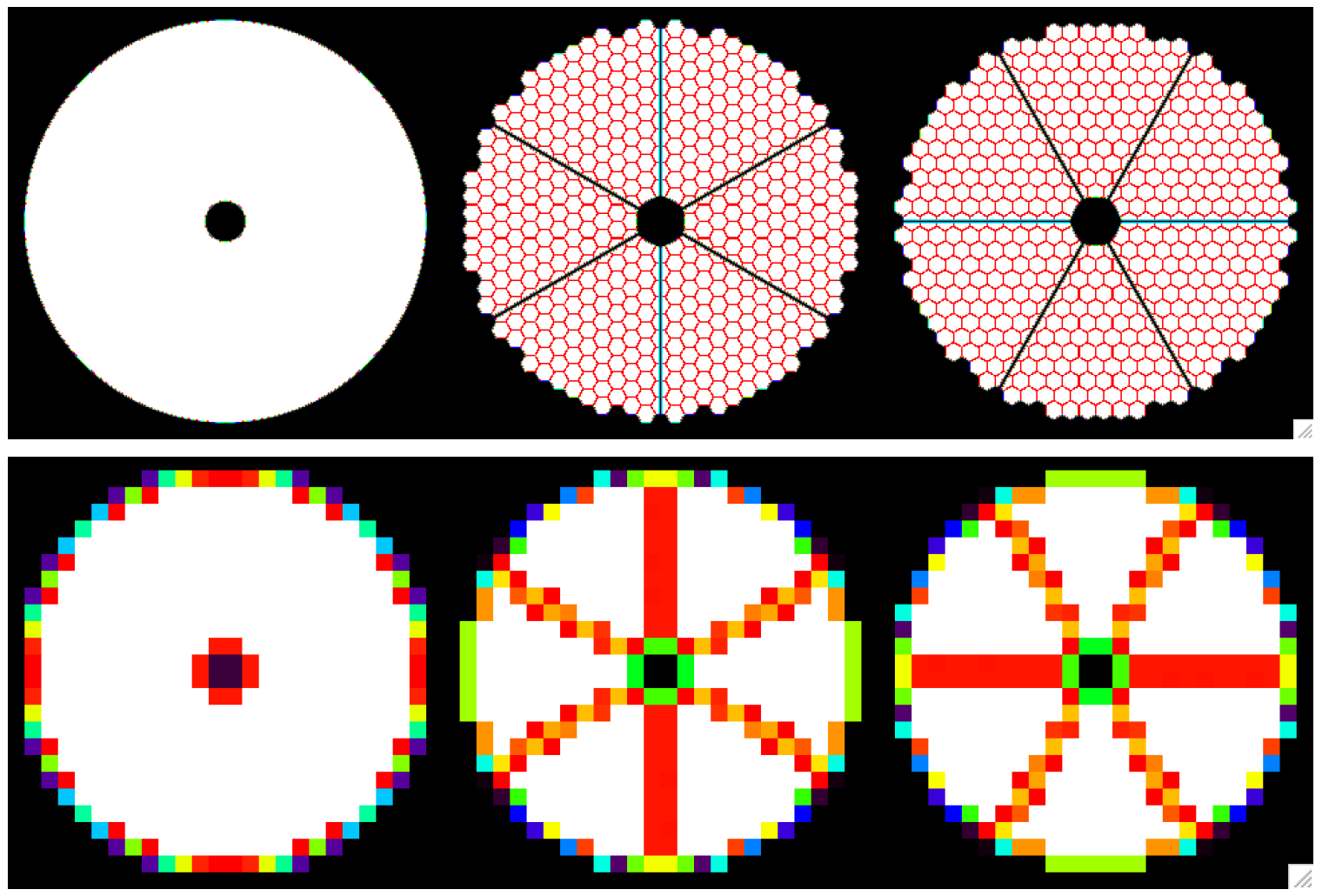

Figure 4: TMT pupils at full resolution (top), and rebinned with 24 pixels across the diameter (bottom)

In normal operation when NFIRAOS operates with LGSs and the NGS WFS is used as a truth sensor, the WFS, which is a PWFS, is binned. The bottom of Figure 4 shows the binned pupil image (obtained by summing the four PWFS pupil images) when 24 pixels are used in the diameter.

\subsection{NFIRAOS PWFS}

NFIRAOS PWFS (Truth WFS) creates four pupil images, which nominally have 96 pixels across the diameter. Several effects combine to blur these images:

- Charge diffusion at the CCD

- Image quality of optical relay

- Diffraction of 2" field stop

- Fast Steering Mirror providing the modulation not exactly at pupil plane

For NFIRAOS, these effects combine to a Gaussian blurring kernel with a full-width at half maximum (FWHM) of about 1 pixel. Figure 5 shows the effect of blurring on the pupil images. 

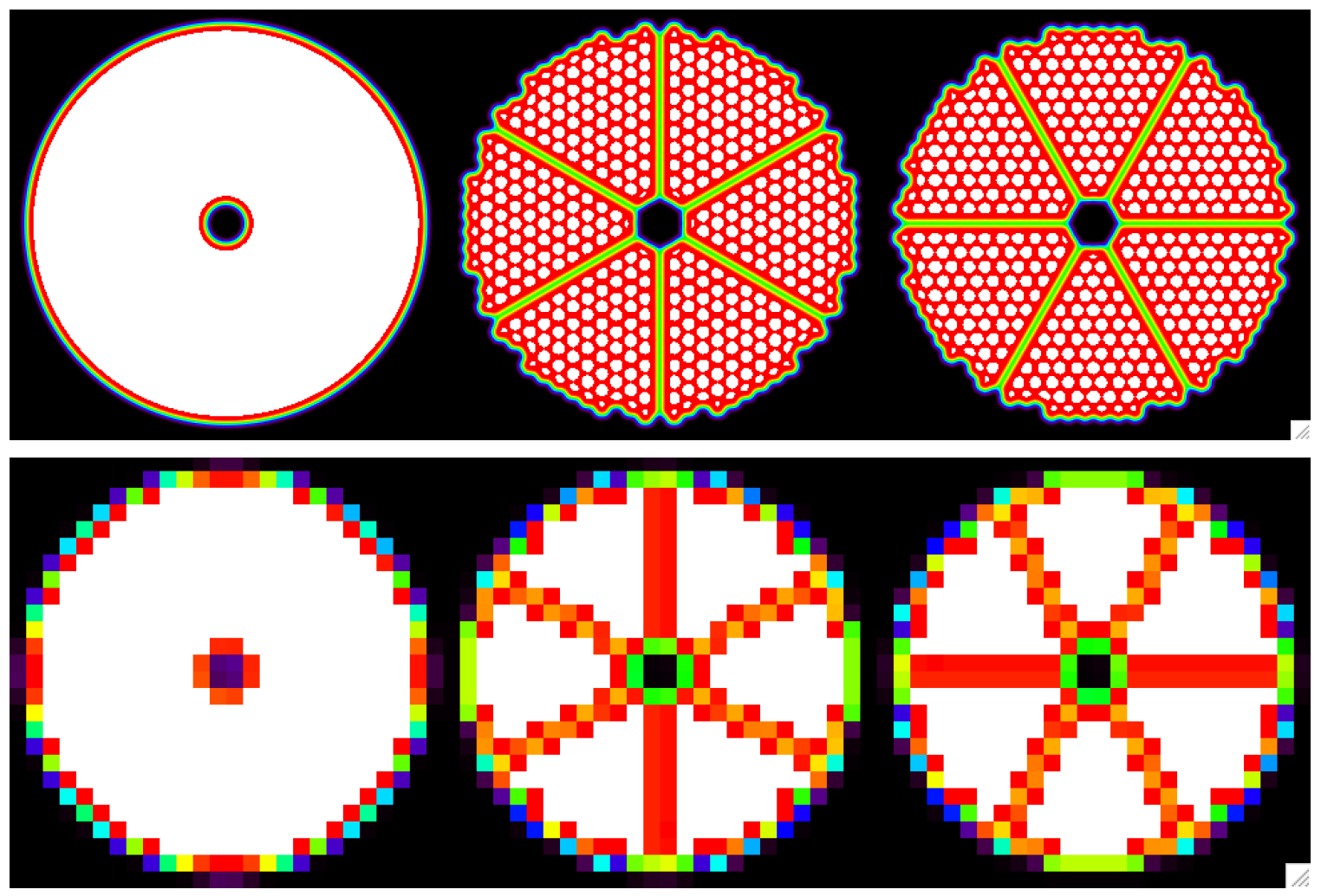

Figure 5: Pupil images at full resolution (top) and with a 24 pixel resolution (bottom), when blurring is included

\subsection{Flux assumptions}

We assume a zero point in $\mathrm{R}$ band, which is the sensing band of the PWFS, of $1.35 \mathrm{e} 10$ photons $/ \mathrm{m}^{2} / \mathrm{s}$. The TMT collecting area is about $707 \mathrm{~m}^{2}$ and the throughput to the PWFS is 0.424 . The background level at $\mathrm{R}$ band is 660,000 photons/s/arcsec ${ }^{2}$ in bright time (full moon) and ten times less in dark time (new moon) The background level on the PWFS images is computed by integrating through a 2 arcsec field stop. The detector is assumed to have 1 electron RMS of noise at the sampling frequencies of interest $<3 \mathrm{~Hz}$. Also, each of the $492 \mathrm{M} 1$ segment will differ in reflectivity depending on the segment recoating schedule. It is expected that the reflectivity of each segment will vary by $\pm 3 \%$ at any given time [2].

\subsection{Pupil position estimation algorithms}

The position of the pupil is estimated from the pupil image obtained from the PWFS. We assume that the PWFS looks at a star with R-magnitude of 18.5 (which is the maximum magnitude of the brightest of the three NGS stars) with an integration time of $0.33 \mathrm{~s} \mathrm{(3} \mathrm{Hz} \mathrm{is} \mathrm{the} \mathrm{maximum} \mathrm{frame} \mathrm{rate} \mathrm{for} \mathrm{the} \mathrm{NFIRAOS} \mathrm{TWFS).} \mathrm{From} \mathrm{the} \mathrm{numbers} \mathrm{in} \mathrm{section} \mathrm{3.4,} \mathrm{it}$ is easy to see that most of the photons actually comes from the sky background, not the star.

We propose to evaluate three different algorithms to estimate pupil position:

- Center of gravity

- Correlation + center of gravity

- Unconstrained matched filter

To build the matched filter, the derivatives can be obtained numerically, using a digital representation of the expected pupil image, or optically using pupil steering mirrors in PWFS path. The matched filters for the $\mathrm{X}$ and $\mathrm{Y}$ directions are shown in Figure 6. 


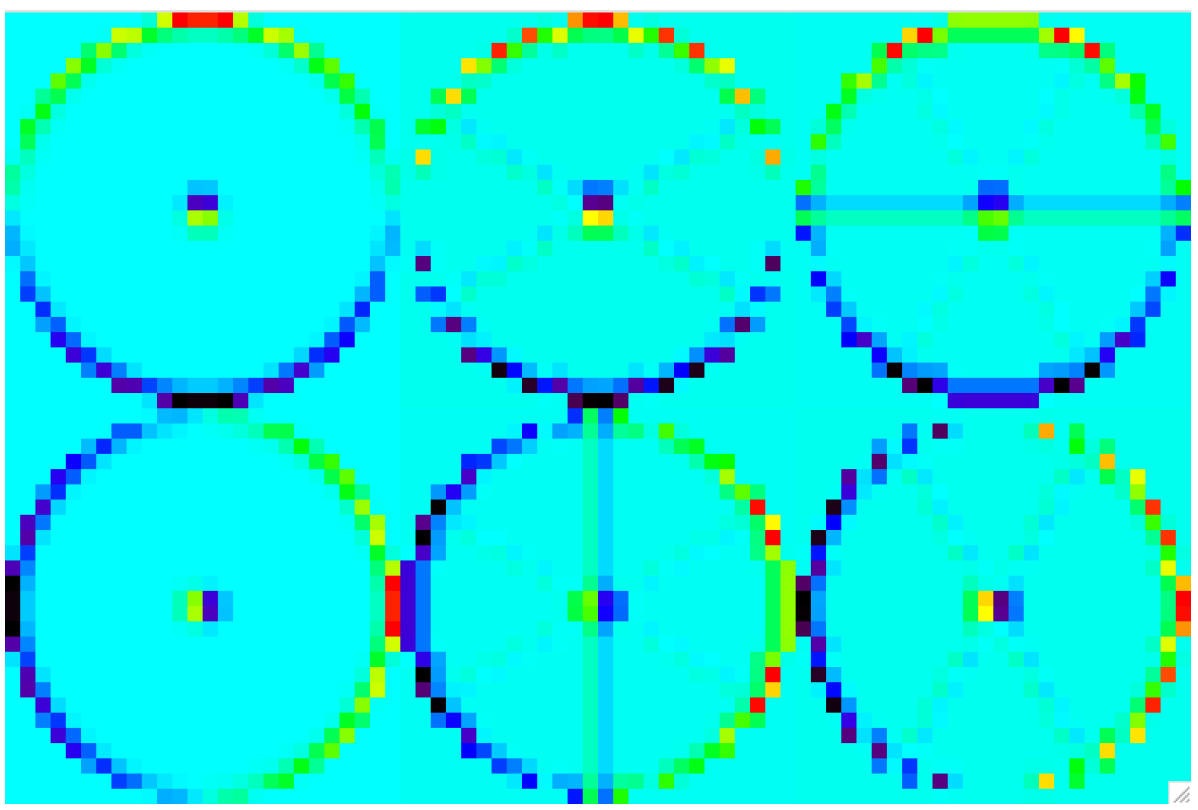

Figure 6: Matched filters for X (top) and Y (bottom) for each of the three TMT pupils under study

\subsection{Diffraction effects on pupil images}

It is well known that diffraction has an important effect on PWFS pupil images. It is therefore important to verify that summing the four pupil images does provide a good estimate of the full pupil image. Figure 7 shows that diffraction has quite an effect on full resolution images, especially at small modulation. However, Figure 8 shows that when the resolution is degraded to 20 pixels across the pupil diameter, the effect is much reduced and negligible when the modulation is greater than $10 \lambda / \mathrm{D}$.

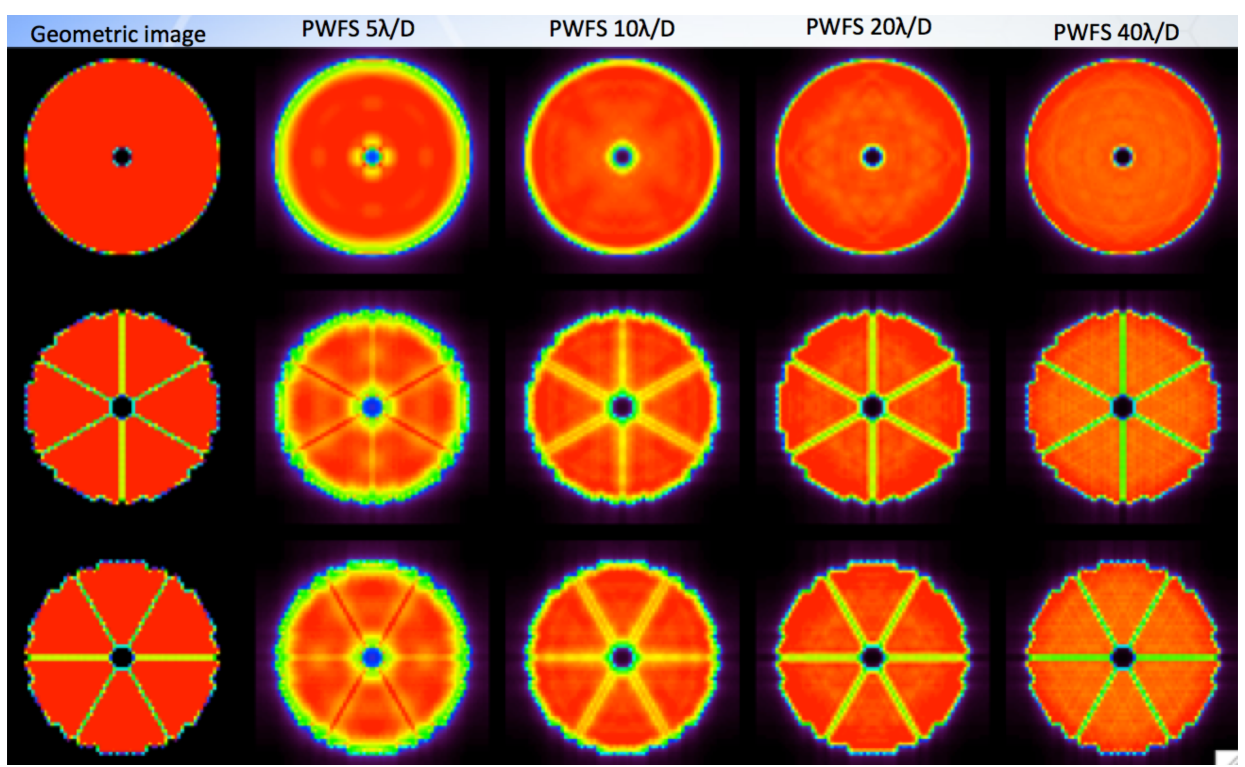

Figure 7: Pupil image estimated geometrically (left column) and using Fourier optics for different modulation radii when the PWFS has a 60x60 resolution 


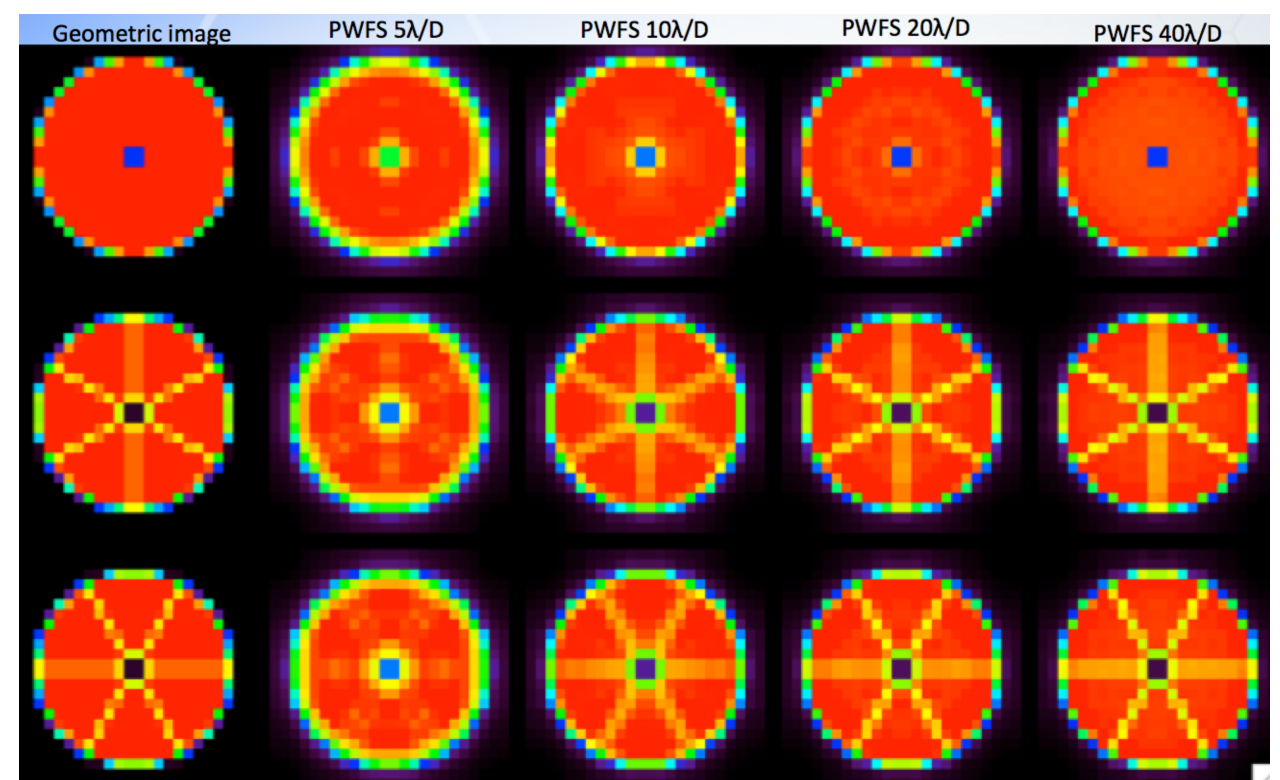

Figure 8: Pupil image estimated geometrically (left column) and using Fourier optics for different modulation radii when the PWFS has a $20 \times 20$ resolution

\subsection{Monte-Carlo simulations and results}

Our Monte-Carlo simulations consist in 10,000 realizations of a pupil image, including photon and read-out noise. For each realization, we use the three different algorithms to estimate the position of the pupil image and compute the position error as the difference between the estimated position and the actual position. Then the RMS of the position error is computed over the 10,000 realizations.

We initially assume that all the M1 segment have the same reflectivity. The results are presented in Table 1 . We see that the centroid and the correlation algorithms provide the same RMS error (probably because the pupil image does not have a peaked shape). Matched filters provide a $\sim \mathrm{x} 3$ reduction in RMS error, and so, with matched filters, the typical RMS error in bright time less than $0.01 \%$ of the pupil diameter.

\begin{tabular}{|c|c|c|c|c|c|c|}
\hline & \multicolumn{2}{|c|}{ Circular pupil } & \multicolumn{2}{c|}{ TMT Pupil 1 } & \multicolumn{2}{c|}{ TMT Pupil 2 } \\
\hline & RMS-x & RMS-y & RMS-x & RMS-y & RMS-x & RMS-y \\
\hline Centroid & $0.0260 \%$ & $0.0259 \%$ & $0.0255 \%$ & $0.0257 \%$ & $0.0256 \%$ & $0.0256 \%$ \\
\hline Correlation & $0.0260 \%$ & $0.0259 \%$ & $0.0255 \%$ & $0.0257 \%$ & $0.0256 \%$ & $0.0256 \%$ \\
\hline Matched filter & $0.0089 \%$ & $0.0089 \%$ & $0.0088 \%$ & $0.0083 \%$ & $0.0083 \%$ & $0.0088 \%$ \\
\hline
\end{tabular}

Table 1: RMS pupil position error (in percent of pupil diameter) for different TMT pupils and different estimation algorithm in bright time, with photon / read-out noise and uniform M1 reflectivity

Still in bright time, we now examine the effect of reflectivity non-uniformity of the M1 segments. We are still performing a Monte-Carlo simulation with 10,000 realizations of a TMT pupil image, but this time, we do not inject any photon / read-out noise. Instead, for each realization, we modify the reflectivity of the M1 segments by $\pm 3 \%$ using a uniform random distribution. This results in a pupil position estimation error, and, as above, we record the RMS of this error over the 10,000 realizations. The results are presented in Table 2. Again, centroid and correlation provide the same RMS error, and matched filters reduce the RMS estimation error by $\sim$ x5. With matched filters, the typical RMS error in bright time is $0.005 \%$ of the pupil diameter. 


\begin{tabular}{|c|c|c|c|c|c|c|}
\hline & \multicolumn{2}{|c|}{ Circular pupil } & \multicolumn{2}{c|}{ TMT Pupil 1 } & \multicolumn{2}{c|}{ TMT Pupil 2 } \\
\hline & RMS-x & RMS-y & RMS-x & RMS-y & RMS-x & RMS-y \\
\hline Centroid & $0.0202 \%$ & $0.0198 \%$ & $0.0204 \%$ & $0.0199 \%$ & $0.0204 \%$ & $0.0199 \%$ \\
\hline Correlation & $0.0202 \%$ & $0.0198 \%$ & $0.0204 \%$ & $0.0199 \%$ & $0.0204 \%$ & $0.0199 \%$ \\
\hline Matched filter & $0.0043 \%$ & $0.0043 \%$ & $0.0048 \%$ & $0.0041 \%$ & $0.0041 \%$ & $0.0046 \%$ \\
\hline
\end{tabular}

Table 2: RMS pupil position error (in percent of pupil diameter) for different TMT pupils and different estimation algorithm in bright time, with no photon / read-out noise but with non-uniform M1 reflectivity

While most $\mathrm{AO}$ observations occur in bright time, it is important to assess how well we can estimate the pupil position in dark time, where the sky background, which provides the majority of the photons in the pupil image, is ten times fainter. We have carried out the same simulations as above for the dark time case and the results are Table 3 and Table 4 . We see that the matched filters provide the same level of improvement as in bright time, however the typical RMS position estimation error due to photon and read-out noise is increased to $\sim 0.022 \%$ of pupil diameter. On the other hand, the RMS position estimation error due to non-uniformity of the reflectivity of M1 remains $\sim 0.005 \%$.

\begin{tabular}{|c|c|c|c|c|c|c|}
\hline & \multicolumn{2}{|c|}{ Circular pupil } & \multicolumn{2}{c|}{ TMT Pupil 1 } & \multicolumn{2}{c|}{ TMT Pupil 2 } \\
\hline & RMS- $x$ & RMS-y & RMS-x & RMS-y & RMS-x & RMS-y \\
\hline Centroid & $0.0669 \%$ & $0.0668 \%$ & $0.0654 \%$ & $0.0661 \%$ & $0.0659 \%$ & $0.0653 \%$ \\
\hline Correlation & $0.0669 \%$ & $0.0668 \%$ & $0.0654 \%$ & $0.0661 \%$ & $0.0659 \%$ & $0.0653 \%$ \\
\hline Matched filter & $0.0221 \%$ & $0.0218 \%$ & $0.0216 \%$ & $0.0205 \%$ & $0.0208 \%$ & $0.0216 \%$ \\
\hline
\end{tabular}

Table 3: RMS pupil position error (in percent of pupil diameter) for different TMT pupils and different estimation algorithm in dark time, with photon / read-out noise and uniform M1 reflectivity

\begin{tabular}{|c|c|c|c|c|c|c|}
\hline & \multicolumn{2}{|c|}{ Circular pupil } & \multicolumn{2}{c|}{ TMT Pupil 1 } & \multicolumn{2}{c|}{ TMT Pupil 2 } \\
\hline & RMS-x & RMS-y & RMS-x & RMS-y & RMS- $x$ & RMS-y \\
\hline Centroid & $0.0202 \%$ & $0.0198 \%$ & $0.0204 \%$ & $0.0199 \%$ & $0.0204 \%$ & $0.0199 \%$ \\
\hline Correlation & $0.0202 \%$ & $0.0198 \%$ & $0.0204 \%$ & $0.0199 \%$ & $0.0204 \%$ & $0.0199 \%$ \\
\hline Matched filter & $0.0040 \%$ & $0.0040 \%$ & $0.0045 \%$ & $0.0038 \%$ & $0.0039 \%$ & $0.0044 \%$ \\
\hline
\end{tabular}

Table 4: RMS pupil position error (in percent of pupil diameter) for different TMT pupils and different estimation algorithm in dark time, with no photon / read-out noise but with non-uniform M1 reflectivity

\subsection{Use of a unique matched filter}

In the previous section, we have seen that the matched filters provide significant improvement compared to the center of gravity and correlation algorithms. However, they need to be recomputed as the image of the TMT pupil continuously rotates on the PWFS, to take into account the proper orientation of the ragged edges and spider. In this section, we ask ourselves whether a matched filter computed for the simple annular aperture can be successfully used to estimate the position of the TMT pupil. We first compute the response of the "Circular (annular) pupil" matched-filter when applied on "TMT Pupil 1". The results are shown in Figure 9. We see that in order to use a matched-filter built with an annular pupil we need to multiply its output by 1.8 in order to match the response of a matched-filter computed with the actual "TMT Pupil 1". 


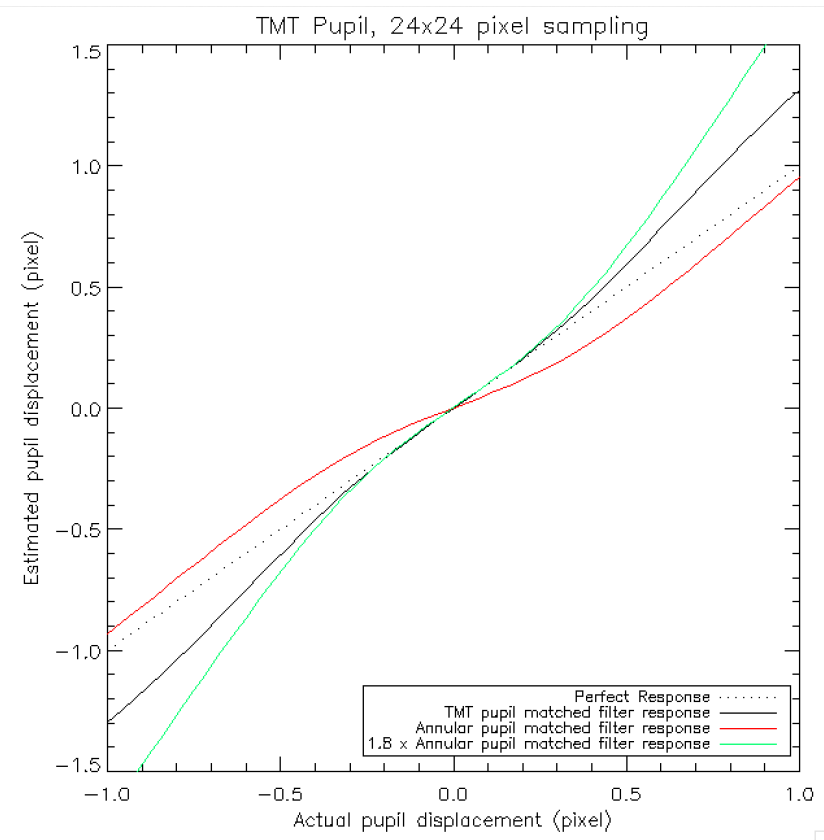

Figure 9: Response of matched filters obtained with different pupil images compared to ideal response (dotted line)

Once this adjustment has been made we can rerun the four sets of simulations from section 3.7. Now the matched filters are all identical and built from the annular pupil. The results, for the dark time case, are given in Table 5 and Table 6 . These results can be compared directly to those in Table 3 and Table 4 to understand the effect of using a generic matched filter for all pupil rotation (the results for the "Circular pupil" are of course the same). We see that the typical RMS error due to noise is increased to $\sim 0.028 \%$ of the pupil diameter, while the RMS error due to the non-uniformity of the M1 reflectivity remains about the same at $\sim 0.005 \%$ of the pupil diameter.

\begin{tabular}{|c|c|c|c|c|c|c|}
\hline \multirow{2}{*}{} & \multicolumn{2}{|c|}{ Circular pupil } & \multicolumn{2}{c|}{ TMT Pupil 1 } & \multicolumn{2}{c|}{ TMT Pupil 2 } \\
\hline & RMS-x & RMS-y & RMS-x & RMS-y & RMS-x & RMS-y \\
\hline Centroid & $0.0669 \%$ & $0.0668 \%$ & $0.0654 \%$ & $0.0661 \%$ & $0.0659 \%$ & $0.0653 \%$ \\
\hline Correlation & $0.0669 \%$ & $0.0668 \%$ & $0.0654 \%$ & $0.0661 \%$ & $0.0659 \%$ & $0.0653 \%$ \\
\hline Matched filter & $0.0221 \%$ & $0.0218 \%$ & $0.0247 \%$ & $0.0275 \%$ & $0.0277 \%$ & $0.0248 \%$ \\
\hline
\end{tabular}

Table 5: RMS pupil position error (in percent of pupil diameter) for different TMT pupils and different estimation algorithm in dark time, with photon / read-out noise and uniform M1 reflectivity. All matched filter are built using the circular (annular) pupil.

\begin{tabular}{|c|c|c|c|c|c|c|}
\hline & \multicolumn{2}{|c|}{ Circular pupil } & \multicolumn{2}{c|}{ TMT Pupil 1 } & \multicolumn{2}{c|}{ TMT Pupil 2 } \\
\hline & RMS-x & RMS-y & RMS-x & RMS-y & RMS-x & RMS-y \\
\hline Centroid & $0.0202 \%$ & $0.0198 \%$ & $0.0204 \%$ & $0.0199 \%$ & $0.0204 \%$ & $0.0199 \%$ \\
\hline Correlation & $0.0202 \%$ & $0.0198 \%$ & $0.0204 \%$ & $0.0199 \%$ & $0.0204 \%$ & $0.0199 \%$ \\
\hline Matched filter & $0.0037 \%$ & $0.0036 \%$ & $0.0040 \%$ & $0.0040 \%$ & $0.0040 \%$ & $0.0039 \%$ \\
\hline
\end{tabular}

Table 6: RMS pupil position error (in percent of pupil diameter) for different TMT pupils and different estimation algorithm in dark time, with no photon / read-out noise but with non-uniform M1 reflectivity. All matched filter are built using the circular (annular) pupil. 


\section{CONCLUSION}

By viewing actuator pokes from the NFIRAOS high-altitude DM (DM11) on the IRIS pupil viewing camera, we are able to align the IRIS Lyot mask to the NFIRAOS ground-conjugated DM (DM0) with a very high precision. By viewing actuators pokes from DM0 on the NFIRAOS Truth WFS (Pyramid WFS), we are able to register DM0 and the Truth WFS with a very high precision. These two calibration steps allow us to track the position of the TMT pupil image on the IRIS Lyot stop by processing the Truth WFS images, and feed back any error to the telescope control system to repoint the beam sent to NFIRAOS and correct the pupil position error onto the Lyot stop. Using matched filters to estimate the pupil position from the TWFS images results in significant higher accuracy than using algorithms such as center of gravity and correlation, and we have found that using a generic matched filter valid for all pupil orientations as opposed to a specific matched filter for each orientation results only in a slight increase of the estimation error. Overall, we are able to estimate the pupil position with an error $<0.02 \%$ of the pupil diameter in bright time $(<0.03 \%$ in dark time) on a magnitude 18.5 star and an integration time of $0.33 \mathrm{~s}$. This plays a significant role in ensuring that IRIS can

have a Lyot stop with minimal under-sizing (only 1\%), as discussed in reference [1], and therefore achieve a very high scientific efficiency.

\section{REFERENCES}

[1] Herriot, G., et al., "Point Source sensitivity, Pupil alignment, Calibration and Control for TMT-NFIRAOS-IRIS", this conference

[2] L. Stepp, private communication, 2016 